In der Rubrik „Literatur kompakt" werden die wichtigsten Originalarbeiten aus der internationalen Fachliteratur referiert.

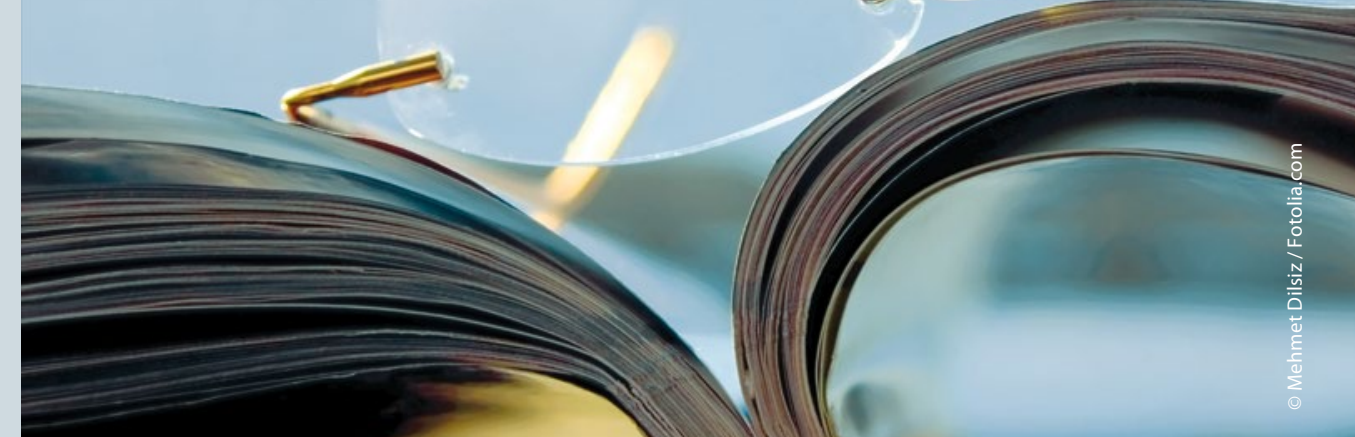

\section{Chronische Rhinosinusitis erhöht Risiko für Cholesteatom}

\section{Patienten mit chronischer Rhinosinusitis haben ein erhöhtes Risiko für ein Cholesteatom. Diesen Zusammenhang lassen die Ergebnisse einer bevölke- rungsgestützten Studie vermuten, in der Befunde von fast 13.000 Patienten ausgewertet worden sind.}

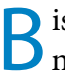
isher gab es nur kleine Studien, in denen die Assoziation zwischen chronischer Rhinosinusitis und Cholesteatom überprüft worden ist. Ärzte um Dr. ChinLung Kuo von der National Yang-Ming University in Taipei in der Republik China haben deshalb die umfangreichen $\mathrm{Da}$ ten der Longitudinal Health Insurance Database of Taiwan ausgewertet, die zwischen 1997 und 2002 erhoben worden waren. Sie stammen von 12.670 Patienten mit auf der Basis des ICD-9-CM neu diagnostizierter chronischer Rhinosinusitis sowie von 63.350 Teilnehmern ohne die Erkrankung. Das Follow-up betrug acht Jahre.
In diesem Zeitraum entwickelten den Ärzten zufolge insgesamt 209 Studienteilnehmer ein Cholesteatom, und zwar 66 in der Gruppe mit Rhinosinusitis (davon fünf Kinder bis 17 Jahre) und 143 in der Kontrollgruppe (davon sieben Kinder). Das entspricht insgesamt Inzidenzraten von 0,65 versus 0,23 pro $1.000 \mathrm{~Pa}$ tientenjahre. Die Inzidenz ist damit in der Rhinosinusitisgruppe mehr als doppelt so hoch. Die höhere Rate ist dabei unabhängig vom Alter, Geschlecht und Follow-up-Dauer.

Die Wahrscheinlichkeit, innerhalb der acht Jahre ein Cholesteatom zu entwi-

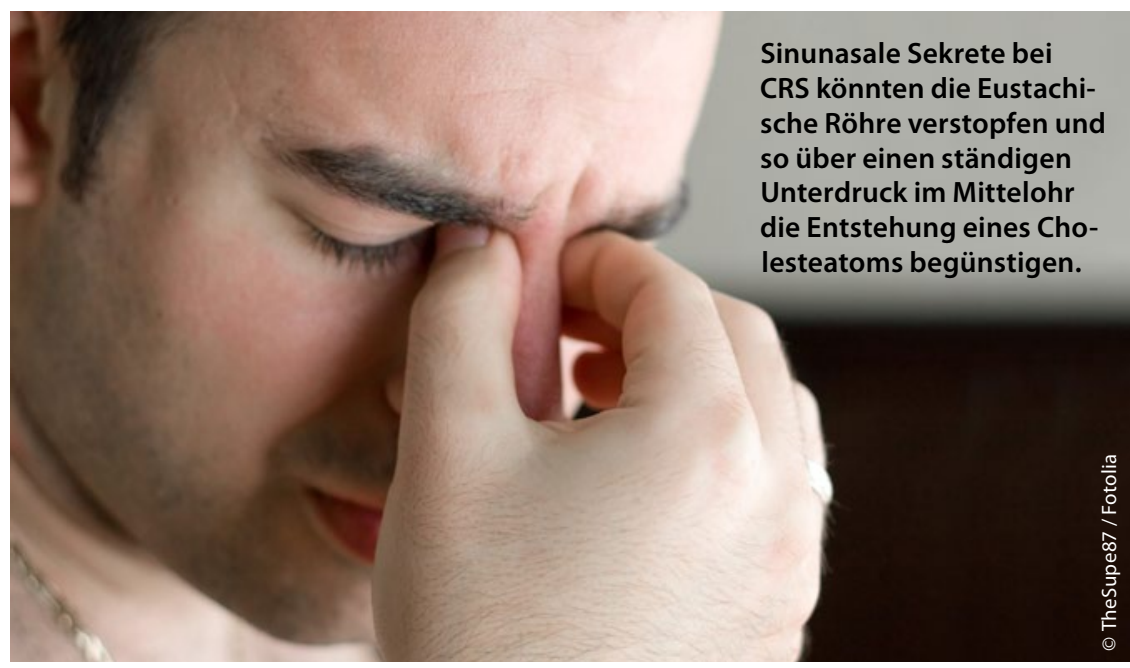

ckeln, war nach Angaben der Ärzte bei Patienten mit chronischer Rhinosinusitis um $69 \%$ erhöht (Hazard Ratio [HR]: 1,69; $95 \%$-Konfidenzintervall zwischen 1,23 und 2,32). Bei der Berechnung dieses Risikos berücksichtigten sie eine ganze Reihe potentiell verzerrender Faktoren wie Asthma, allergische Rhinitis, atopische Dermatitis, Tonsillektomie sowie Alter und Geschlecht. Das höchste Risiko für ein Cholesteatom errechneten die Wissenschaftler bei einem Follow-up von drei Jahren (HR: 2,62; $95 \%$-Konfidenzintervall zwischen 1,65 und 4,17). Später nahm das Risiko kontinuierlich ab.

Wie es in der aktuellen deutschen S-1-Leitlinie zum Cholesteatom von 2014 heißt, ist es definiert als ektopes, verhornendes Plattenepithel in den Mittelohr- und Mastoidräumen. „Charakteristisch ist die langsam fortschreitende, in der Regel schmerzfreie, knochendestruierende Ausbreitung infolge andauernder Entzündungsreaktionen." Dabei könnten der Entzündungsgrad, das Ausmaß der Knochendestruktion und die Ausbreitungsform stark variieren.

Fazit: Die chinesischen Ärzte erklären sich die Entstehung eines Cholesteatoms aufgrund bisheriger Erkenntnisse damit, dass sinunasale Sekrete über die Eustachische Röhre transportiert werden und diese mit der Zeit verstopfen. Dadurch herrsche im Mittelohr ein konstanter Unterdruck, in dessen Folge sich ein Cholesteatom entwickle. Auch die Beteiligung eines Biofilms an der Pathogenese wird diskutiert.

Peter Leiner

Kuo CL et al. Association Between Middle Ear Cholesteatoma and Chronic Rhinosinusitis. JAMA Otolaryngol Head Neck Surg 2017, https:// doi.org/10.1001/jamaoto.2017.0130 